automated features. Both online diary and smartphone application will be demonstrated.

Conclusion Web-based tools to monitor adherence to PrEP within trials are a novel and promising approach, with both advantages (eg. provides a large amount of data) and disadvantages (eg. limited validity). User-participation is an important pre-requisite to tailor such tools to target their needs.

\section{P4.92 START OF A SYNDEMIC BASED INTERVENTION TO FACILITATE CARE FOR MEN WHO HAVE SEX WITH MEN WITH HIGH RISK BEHAVIOUR: THE SYN.BAS.IN RANDOMISED CONTROLLED TRIAL}

${ }^{1}$ Roel Achterbergh, ${ }^{2}$ JJ Van Der Helm, ${ }^{3} \mathrm{~W}$ Van De Brink, ${ }^{4} \mathrm{AA}$ Matser, ${ }^{5} \mathrm{HJC}$ De Vries. ${ }^{1}$ Public Health Serice Amsterdam, Amsterdam, The Netherlands; ${ }^{2}$ Centre for Environmental Safety and Security, National Institute of Public Health and the Environment, Bilthoven, The Netherlands; ${ }^{3}$ Department of Psychiatry, Academic Medical Centre, Amsterdam, The Netherlands; ${ }^{4}$ Public Health Service Amsterdam, Amsterdam, The Netherlands; ${ }^{5}$ Department of Dermatology, Academic Medical Centre, Amsterdam, The Netherlands

\subsection{6/sextrans-2017-053264.587}

Introduction Men who have sex with men (MSM) constitute a risk group for sexual transmitted infections (STIs), including HIV. Despite counselling interventions, risk behaviour remains high. Syndemic theory holds that psychosocial problems often co-occur, interact and mutually reinforce each other, thereby increasing high risk behaviours and co-occurring diseases. Therefore, if co-occurring psychosocial problems were assessed and treated simultaneously, this might decrease high risk behaviour and disease.

Method An open label randomised controlled trial will be conducted among 150 MSM with high risk behaviour recruited from the STI clinic of Amsterdam. Inclusion criteria are: HIV negative MSM with two STI or PEP treatment in the last 24 months or HIV positive MSM with one STI in the last 24 months. All participants get questionnaires on the following syndemic domains: ADHD (ASRS), depression (HADS), anxiety disorder(HADS), alexithymia (TAS) and sex(SCS, Kalichman) and drug addiction(DUDIT and AUDIT). Participants in the control group receive standard care for one year: STI screenings every three months and motivational interviewing based counselling. Participants in the experimental group receive standard care plus additional questionnaires depending on baseline questionnaire scores followed by feedback and referral to a co-located mental health or addiction service in case of a positive indication according to the additional questionnaires. The primary outcome is help seeking behaviour for mental health problems and/or drug use problems. The secondary outcomes are STI incidence and changes in sexual risk behaviour (i.e. condom use, number of anal sex partners, drug use during sex).

Conclusion This study will provide information on syndemic domains among MSM who show high risk behaviour and on the effect of screening and referral on help seeking behaviour and health (behaviour) outcomes. Inclusion started in September 2016, on 1 December more than 60 MSM were included with this pace baseline data will be available in July 2017.

\section{P4.93 ARE RECTAL DOUCHING AND SHARING DOUCHING EQUIPMENT ASSOCIATED WITH ANORECTAL CHLAMYDIA AND GONORRHOEA? A CROSS-SECTIONAL STUDY AMONG MEN WHO HAVE SEX WITH MEN}

${ }^{1}$ Roel Achterbergh, ${ }^{2} \mathrm{JJ}$ Van Der Helm, 'W Van Den Boom, 'T Heijman, ${ }^{1} \mathrm{I} G$ Stolte, ${ }^{1} \mathrm{MS}$ Van Rooijen, ${ }^{3} \mathrm{HJC}$ De Vries. 'Public Health Serice Amsterdam, Amsterdam, The Netherlands; ${ }^{2}$ Centre for Environmental Safety and Security, National Institute of Public Health and the Environment, Bilthoven, The Netherlands; ${ }^{3}$ Department of Dermatology, Academic Medical Centre, Amsterdam, The Netherlands

\subsection{6/sextrans-2017-053264.588}

Introduction Men who have sex with men (MSM) are at high risk for anorectal chlamydia and gonorrhoea infections. Many MSM use rectal douches in preparation for sex, which might break down the mucosal barrier function and facilitate the acquisition of sexually transmitted infections (STI). We determined whether rectal douching or sharing douching equipment was associated with anorectal chlamydia and gonorrhoea.

Methods In a cross-sectional study among 994 MSM attending the STI outpatient clinic of Amsterdam between February and April 2011, data was collected on rectal douching, sexual behaviour, and STI. We used multivariable logistic regression analysis to determine the association between rectal douching, including sharing of douching equipment, and anorectal chlamydia and gonorrhoea for those at risk. We adjusted for other risk behaviour, i.e., condom use, number of partners, and HIV status.

Results Of 994 MSM, 46\% ( $=460)$ practised rectal douching, of whom 25\% ( $n=117)$ shared douching equipment. Median age was 39 years [interquartile range (IQR), 30-47], median number of sex partners in the six months prior to consult was five [IQR 3-10] and 289 (29.0\%) participants were HIVpositive. The prevalence of anorectal chlamydia and/or gonorrhoea for those at risk was 9.6\% $(\mathrm{n}=96)$. In multivariable analysis, $\mathrm{HIV}$ positivity $(\mathrm{aOR}=2.2,95 \% \mathrm{CI}=1.3-3.6)$, younger age $(\mathrm{aOR}=2.5, \mathrm{CI}=1.4-4.5$ for those $<35$ years compared to $\geq 45$ years), and more sexual partners $(\mathrm{aOR}=1.2 ; 95 \%$ $\mathrm{CI}=1.0-1.5$ for $1 \mathrm{log}$ increase) were significantly associated with anorectal STI. However, rectal douching or sharing douching equipment were not significantly associated with anorectal chlamydia and/or gonorrhoea $(p=0.726)$.

Conclusion Almost half of MSM used rectal douching and a quarter of these shared douching equipment. Though using douching equipment does not appear to contribute to anorectal chlamydia and gonorrhoea, STI prevalence remains high and prevention strategies like early testing and treatment remain of utmost importance.

\section{P4.94 STI RATES AMONG HOMELESS PERSONS IN THE U.S}

${ }^{1}$ Samantha P Williams, ${ }^{1}$ Alexandra E Caccamo, ${ }^{1}$ Rachel Kachur, ${ }^{2}$ Kenneth L Bryant. ${ }^{1}$ Division of STD Prevention, Centres for Disease Control and Prevention, Atlanta, USA; ${ }^{2}$ Division of Global HIV and TB, Centres for Disease Control and Prevention, Atlanta, USA

\subsection{6/sextrans-2017-053264.589}

Introduction Homelessness is a societal problem with public health implications. The U.S rate of homelessness is 17.7/ 10,000. Nightly, 550000 persons experience homelessness, $6.5 \%$ under the age of 18 years. Homelessness is associated with greater engagement in high-risk sexual behaviour. Less is known about sexually transmitted infections (STI) among the homeless. 
Methods We systematically identified peer-reviewed articles, published 2000-2016, which reported STI bio-specimen testing and rates among homeless persons in the US. Articles $(n=9)$ were grouped by age (e.g. youth $=5$; adults $=4$ ); STI rates and correlate factors were described by gender.

Results Across STIs, rates varied from $0.4 \%-17 \%$ among homeless youth $(\mathrm{n}=5)$, and $0.9 \%-52.5 \%$ among homeless adults $(n=4)$. Rates for chlamydia (CT) were similar for youth $(4.2 \%-11.6 \%) \%)$ and adults (4.6\%-10.8). For gonorrhoea, the highest rates for youth $(0.4 \%-9.9 \%)$ were double those for adults $(0.9 \%-4.1 \%) \%)$. STI rates also varied by age and gender. CT was highest among young females (6.45\%-11.0\%\%); followed by adult females (6.7\%); then young males $(4.73 \%-$ $4.8 \%)$. Rates of HSV-2 (5.52\%-11.85\%\%), HBV (1.42\%$17 \%)$ and trichomoniasis $(2.3 \%)$ were reported only in studies on youth. HCV varied among youth (3.77\%-12\%)\%) and adults $(9.8 \%-52.5 \% \%)$, with rates highest among homeless adult men. Composite STI rates were most often reported for adults (16.4\%-40.7\%\%), with highest rates among adult women who experienced intimate partner violence (IPV) (25.9\%-40.7\%)\%).For youth, longer periods of homelessness were associated with high-risk sexual behaviours. Peer social networks had protective and risk effects on sexual behaviour. For adults, frequency and length of homelessness, incarceration history, substance use, injection drug use (IDU), and IPV were associated with having an STI.

Conclusion STI rates among homeless persons varied by age and gender. There is a need for more complete STI data on homeless youth and adults. Such data can aid in understanding STI risk while homeless and how best to develop tailored prevention strategies.

\section{P4.95 DECLINING TRENDS IN RISK BEHAVIOURS AND HIVISTIS AMONG INJECTING DRUG USERS IN INDIA: FINDINGS FROM LARGE SCALE BIO-BEHAVIOURAL SURVEYS FROM THREE HIGH PREVALENT STATES}

Santosh Kumar Sharma. International Institute For Population Sciences, Mumbai, India

\subsection{6/sextrans-2017-053264.590}

Introduction Injecting Drug Users in India are at the duel risk of needle sharing and risky sexual behaviour, becoming more vulnerable to STI and HIV. Although injecting drug users constitute a risk group in themselves, there is also an overlap between drug addiction and those involved in sex work. Individuals who fall into both categories are therefore particularly vulnerable to HIV and are perhaps doubly stigmatised. Thus, studying the interface of injecting drug use and risky sexual behaviour is important to curb the pace of STI/HIV epidemic among IDUs.

Methods Data were obtained from two rounds of integrated biological and behavioural assessments (IBBA) conducted in 2006-07 and 2009-10 where 2,075 IDUs in round-1 and 1977 in round-2 were sampled. Data from both rounds were pooled. Bivariate and multivariate analysis were used for the study.

Results Data from 4052 IDUs from 6 districts of Manipur, Nagaland, and Maharashtra, were analysed. The mean age of IDUs in round- 1 was 26 years and 29 years in round-2. The majority of the IDUs were illiterate, unmarried, employed in two consecutive rounds. IDUs's duration between first drug use to first injecting drug use $\geq 2$ years were 14 times higher during round-2. HIV and any STI symptoms in the round-2 show the declining pattern. IDUs having the main regular partner and shared needle/syringe were less likely to consistency in condom use with different type of female partners across the rounds. Logistics regression analysis of experienced any STI symptom in the past 1 year indicate that IDUs, literate $(\mathrm{AOR}=1.878, \mathrm{p}<0.10)$, age at first injecting drugs 26 and above $(\mathrm{AOR}=2.192, \mathrm{p}<0.05)$ and sharing needle/syringes in the past month $(\mathrm{AOR}=2.218, \mathrm{p}<0.01)$ were significantly more likely than their counterparts. IDUs, $\geq 30$ years (15.951, $\mathrm{p}<0.01)$, literate $(3.415, \mathrm{p}<0.01)$ and shared needle/syringes in the past one month were significantly more likely to have HIV.

Conclusions Harm reduction and needle exchange program should also focus on the risky behaviour of injecting drug users through peer-based approach.

\section{P4.96 STIGMATISED BEHAVIOURAL ASPECTS TOWARDS MSM DIRECTING PERVASIVE NATURE OF ALCOHOL USE IN ANDHRA PRADESH, INDIA}

Santosh Kumar Sharma. International Institute For Population Sciences, Mumbai, India

\subsection{6/sextrans-2017-053264.591}

Introduction Men who have sex with men (MSM) in India are a core risk group for HIV. Heavy alcohol consumption is associated with increased sexual risk-taking behaviours in many cultures, in particular among MSM. This study illustrates the prevalence and correlates of Alcohol use among MSM by their sociodemographic, sexual risk and other characteristics in Andhra Pradesh, India.

Methods Data from a Behavioural Tracking Survey 2014 conducted with key populations MSM in undivided Andhra Pradesh state in India was used. The survey was conducted in the three districts (Warangal, Karim Nagar, and Nalgonda). A stratified random sampling method used to select the MSM for the survey. The total 1200 MSM were selected for the study. Descriptive statistics (proportions) and bivariate analysis were used to describe the strength and association of MSM' socio-demographic/behavioural characteristics and the dependent variable. For the bivariate analysis, the p-value was calculated by using the chi-square test. Binary logistics regression was used to determine the factor associated with ever consumption of alcohol among MSM.

Results In Andhra Pradesh, of the 1200 MSM who were interviewed, around 69\% of MSM had ever used alcohol. Approximately 31 percent MSM reported that they had experienced discrimination at public places and 11.2 percent of said that they are mentally depressed. There is the significant association between ever used of alcohol and sociodemographic, sexual risk and other characteristic $(p<0.05)$. Multivariate logistics regression demonstrated that currently married $(\mathrm{AOR}=1.6 \mathrm{p}<0.05)$, Panthi MSM (AOR1.7 $\mathrm{p}<0.05)$, wanted to experiment $(\mathrm{AOR}=2.0 \mathrm{p}<0.01)$, was attracted towards MSM $(A O R=2.9 \mathrm{p}<0.01)$, consistent condom use $(\mathrm{AOR}=1.7$ $\mathrm{p}<0.05$ ). High discrimination in Public places $(\mathrm{AOR}=1.4$ $\mathrm{p}<0.05)$. It is also reported that MSM who were in any depression, 2.1 times more likely to be affected by alcohol consumption.

Conclusions HIV-related prevention Programme among MSM in India should also focus on alcohol use and related problems in multilevel context. 


\section{P4.97 UPTAKE OF, AND BARRIERS AND FACILITATORS TO, HIV TESTING IN AUSTRALIAN- AND VIETNAMESE-BORN ADULTS IN QUEENSLAND, AUSTRALIA}

${ }^{1}$ Sarah Blondell, ${ }^{2}$ Mark Griffin, ' Jo Durham. ${ }^{1}$ The University of Queensland, Brisbane, Australia; ${ }^{2}$ Adasis, Brisbane, Australia

\subsection{6/sextrans-2017-053264.592}

Introduction In Australia, people born in South-East Asia have the highest proportion of late human immunodeficiency virus (HIV) diagnosis. Late diagnosis has important implications for prevention, treatment and management of HIV. Limited evidence is available, however, on the testing behaviours of the South-East Asian migrant population and general Australian population.

Methods The study used quantitative and qualitative methods to assess uptake of, and barriers and facilitators to, HIV testing in Vietnamese- and Australian-born adults (18-49 years) in Queensland, Australia. A 66- item self-administered survey was given to participants (Vietnamese: $n=188$ and Australian: $\mathrm{n}=195$ ) at selected sites in Brisbane using convenience sampling. The survey included demographic, HIV knowledge, HIV stigma, HIV risk, HIV testing and healthcare access items. Materials were available in Vietnamese and English. Qualitative interviews $(n=8)$ were done with Vietnamese-born survey participants to further explore survey findings.

Results No statistically significant differences were seen between the two groups in terms of ever tested, ever recommended a test, self-testing or same day testing. Vietnameseborn people were significantly more likely to have recently tested and to accept provider-initiated testing and counselling (PITC). Interview data also suggested a preference for PITC in the Vietnamese-born community, due to their trust in doctors. They also indicated potential high-risk sexual practices in Vietnamese-born men.

Conclusion The Australian- and Vietnamese-born populations had similar HIV testing practices. Preference for PITC, however, was greater in the Vietnamese-born population. PITC may help reduce late HIV diagnosis by overcoming a number of barriers associated with client-initiated testing, e.g. poor risk perception and knowledge. Further research on the preference for PITC in this population and with healthcare providers who would be offering PITC is needed. Additionally, research is needed into the risk of HIV exposure when travelling back to Vietnam.

\section{P4.98 SPIRO, A PATIENT-MANAGED MOBILE APPLICATION TO IMPROVE SYPHILIS TREATMENT FOLLOW-UP AMONG MEN WHO HAVE SEX WITH MEN}

${ }^{1}$ Sebastiaan Hulstein, ${ }^{1}$ Menne Bartelsman, ${ }^{1}$ Freke Zuure, ${ }^{1}$ Udi Davidovich, ${ }^{1}$ Amy Matser, ${ }^{1}$ Arjan Hogewoning, ${ }^{2}$ Henry DE Vries. ${ }^{1}$ Public Health Service of Amsterdam (GGD Amsterdam), Amsterdam, The Netherlands; ${ }^{2}$ Academic Medical Centre, Department of Dermatology, Amsterdam, The Netherlands

\subsection{6/sextrans-2017-053264.593}

Introduction Syphilis incidence is rising in developed countries worldwide, and men who have sex with men (MSM) are disproportionally affected. Follow-up after treatment of syphilis is important as late complications (e.g. neurosyphilis), therapy failure and re-infections can occur, especially in HIV coinfected patients. Failure to complete follow-up occurs frequently. Moreover, medical history at follow-up is often incomplete, as follow-up occurs often at various health care providers. Self-management of the relevant medical data might improve patient engagement, and ensure the availability of medical data required during follow-up visits.

Methods We developed a patient-managed mobile phone application - Spiro - that can be used by patients treated for syphilis, to record relevant data for follow-up like medical history, physical findings, treponomal (e.g. TTPA) and non-treponomal (e.g. VDRL) serum antibody results, conclusions and treatment. Spiro automatically summarises the data relevant for syphilis follow-up including a graphical depiction of the nontreponomal serum antibody titer over time. Follow-up data are stored locally on the mobile device. Optionally, encrypted online storage is available. Lay information on risk factors for, and symptoms, diagnosis and treatment of syphilis infections is available via the app.

Results After launching Spiro on World AIDS day 2016, we intend to measure its acceptability and usability among patients who use the app and their care providers. In addition, we plan to evaluate retention to follow-up, the availability of required data for syphilis treatment follow-up, healthliteracy and self-reported level of disease control among MSM newly diagnosed with syphilis in a multi-centre RCT, comparing standard care plus the use of Spiro by patients to standard care alone.

Conclusion If feasible and effective, Spiro, a newly developed medical mobile phone application, can improve the engagement in, and retention to syphilis treatment follow-up among MSM.

\section{P4.99 HIV STATUS DOES IT MAKE A DIFFERENCE? SEXUAL BEHAVIOUROF HIV POSITIVE MEN ATTENDING A COMPREHENSIVE TREATMENT CENTRE IN LAGOS, NIGERIA}

${ }^{1}$ Sekoni Adekemi, ${ }^{1}$ Uthman Oluwaseun, ${ }^{2}$ Somefun Esther. 'University of Lagos, Lagos, Nigeria; ${ }^{2}$ Lagos University Teaching Hospital, Lagos, Nigeria

\subsection{6/sextrans-2017-053264.594}

Introduction In many sub-Saharan African countries, most new cases of Human Immunodeficiency Virus (HIV) infection occur in HIV sero-discordant couples. This study assessed the sexual behaviour of HIV positive men accessing comprehensive services at Nigerian Institute of Medical Research Lagos.

Methods A cross sectional study was carried out among men accessing ARV at a PEPFAR Clinic. Ethical approval was obtained. Respondents were recruited consecutively on clinic days among the attendees until calculated sample size of 384 was attained. Data was analysed using IBM SPSS version 20. Association was explored using $\mathrm{p}<0.05$.

Results The mean age was 43.06 years. $74.8 \%$ were married at diagnosis, $58.1 \%$ tested with their partner, $78.5 \%$ knows the HIV status of their partners and $79.2 \%$ had been on ARV for more than a year. With respect to sexual behaviour, $38.9 \%$ and $34.3 \%$ engage in transactional and intergenerational sex respectively. $21.1 \%$ and $8.3 \%$ were into concurrent multiple sexual partnerships in the twelve months and three months preceding the survey. $7.2 \%$ had sex with casual partners or sex workers, $45.4 \%$ did not use condom consistently, among these men, $18.1 \%$ did not use condom at last sex and the last sexual partner for $7.9 \%$ was a sex worker/casual friend. Unmarried respondents were more likely to have 